\title{
Walking Cautiously Into the Collatz Wilderness: Algorithmically, Number Theoretically, Randomly
}

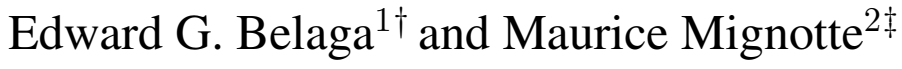 \\ ${ }^{1}$ Institut de Recherche Mathématique Avancée de Strasbourg, 7 rue René Descartes, F-67084 Strasbourg Cedex, \\ FRANCE \\ ${ }^{2}$ Département de Mathématique, Université Louis Pasteur, 7 rue René Descartes, F-67084 Strasbourg Cedex, FRANCE
}

Building on theoretical insights and rich experimental data of our preprints, we present here new theoretical and experimental results in three interrelated approaches to the Collatz problem and its generalizations: algorithmic decidability, random behavior, and Diophantine representation of related discrete dynamical systems, and their cyclic and divergent properties.

Keywords: $3 n+1$, or Collatz problem, Collatz and Conway transforms, discrete dynamical systems, pseudo-rundom walks, algorithmic decidability, exponential Diophantine equations, Diophantine approximations

\section{Introduction}

From many points of view, the challenge posed by the Collatz problem - written down in his notebook by Lothar Collatz in 1937 and known today also as the $3 n+1$ mapping, $3 n+1$ problem, Hasse's algorithm, Kakutani's problem, Syracuse algorithm, Syracuse problem, Thwaites conjecture, and Ulam's problem is unique in the history of modern mathematics.

The fact is - even if the claim of the Collatz conjecture can be easily understood and appreciated by children entering secondary school and in its classic simplicity and beauty it belongs more to the Euclidean than to modern era of mathematics - it remains still unresolved, after more than forty years of sustained theoretical and experimental efforts: cf. the annotated bibliography, by Jeff Lagarias, of 200 papers, books, and preprints [7].

Bibliographical Digression. To avoid bibliographical redundancy, all our references with numbers in outward square brackets direct the reader to corresponding items of the Lagarias bibliography, with his generally very helpful succinct comments. Three papers common to the Lagarias and our own bibliography, at the end of the paper, will be referred to by the double references, as in [3]-]22[, for the 1999 paper of the authors.

Not less surprisingly, the Collatz problem and its immediate elementary generalizations ]21[, ]22[, ]23[, ]34[, ]37[, ]52[, ]53[, ]85[, ]105[, ]116[, ]121[, were shown to be relevant in one or another substantial way to the deepest open questions in algorithmic theory ]20[, ]52[, ]53[, ]98[, ]121[, ]131[, ]155[ and mathematical logic ]56[, ]116[, ]119[, Diophantine approximations and equations theory ]21[, ]22[, ]23[, ]36[, ]133[, ]154[, ]158[, ]159[, ]160[, ]165[, p-adic number theory ]1[, ]27[, ]28[, ]43[, ]137[, ]138$139[$, multiplicative semigroup theory ]64[, ]13 [, ]107[, discrete and continuous dynamical systems theory ]44[, ]59[, ]95[, ]115[, ]134[, ]141[, discrete (random walks) ]32[, ]66, 67[, ]147[, ]182[ and continuous stochastic processes theories ]78[, ]96[, ]97[, ]106[, ]111[, ]112[, ]149[, ]161[, ]162[, ]163[, ]178[, ]181[, ]185[.

The present paper is building on theoretical insights of the short preprint of the first author [2]-]20[ and on rich experimental data presented in our common preprints, [5](vast tables of primitive cycles for 6667 $(3 m+d)$-dynamical systems) and [6] (factorization of Collatz numbers $2^{\ell}-3^{k}$, for all $2 \leq \ell \leq 114,1 \leq$ $k<\frac{\ell}{\log _{2} 3}$,) and interpreted in our preprint [4]-]23[ (long, 62 pages, and dense: cf. the related comment in

\footnotetext{
†belaga@math.u-strasbg.fr

${ }^{\ddagger}$ mignotte@ math.u-strasbg.fr 
Lagarias bibliography), - to succinctly report on our new theoretical and experimental results in three domains closely related to the Collatz problem and its generalizations: algorithmic decidability, asymptotical behaviour of corresponding dynamical systems, and Diophantine interpretation of cyclic properties of such systems.

\section{Basic Definitions, Notations, and Problems}

The Collatz problem concerns the iteration of the Collatz map $T$ defined on the set of natural numbers:

$$
T: \mathbb{N} \longrightarrow \mathbb{N} ; \quad T(n)=\left\{\begin{array}{cl}
\frac{n}{2} & \text { if } n \text { is even; } \\
\frac{3 n+1}{2} & \text { if } n \text { is odd. }
\end{array}\right.
$$

The Collatz conjecture states that a $T$-trajectory starting from any natural number $n$ passes ultimately by 1 (in other words, for some $k>1, T^{k}(n)=1$ ), to become the cyclic trajectory

$$
\mathbf{C}^{o}=\langle 1 \rightarrow 2 \rightarrow 1\rangle .
$$

Since any $T$-trajectory is either divergent or ultimately cyclic (see Definition 2 below), the Collatz conjecture affirms that there exists only one $T$-cyclic trajectory (or $T$-cycle), namely, (2) (the cyclic part of the Collatz conjecture) and that there exist no divergent trajectories (the divergent part of the Collatz conjecture). It goes without saying that both Collatz sub-conjectures are still open.

The Collatz map being defined by the algorithm (1) and, as a result, the Collatz problem being reminiscent of the halting problem for Turing machines, one cannot exclude the unsolvability option to which we now proceed.

Following the trodden path of tweaking with difficult problems by looking at their generalizations, John H. Conway has discovered in 1972 ]52[ a general and, in a sense, the most natural framework for Collatztype problems, the Conway map on $\mathbb{Z}$ (reinvented twice, first, in ]37[ as periodically linear or congruential function, then in ]123[, ]124[, as generalized Collatz map, and extended in [2]-]20[ to piecewise periodically linear functions defined on subsets $\mathbb{Z}_{d}$ of integers relatively prime to $d \geq 2$ ).

Definition 1 Let $p \geq 2$ be a natural number and

$$
M_{p}=\left\{m_{0}, m_{1}, \ldots, m_{p-1}\right\} \in \mathbb{N}^{p}, \quad R_{p}=\left\{r_{0}, r_{1}, \ldots, r_{p-1}\right\} \in \mathbb{Z}^{p}
$$

be two p-tuples of, respectively, natural numbers and integers, such that

$$
\text { for all } i=0, \ldots, p-1, \operatorname{gcd}\left(m_{i}, p\right)=1 \& r_{i} \equiv i m_{i} \quad(\bmod p) .
$$

(1) The corresponding Conway $\left(M_{p}, R_{p}\right)-$ map $T=T_{M_{p}, R_{p}}$ is defined on the set $\mathbb{Z}$ of integers as follows:

$$
T: \mathbb{Z} \longrightarrow \mathbb{Z} ; \quad T(n)=\frac{m_{i} n-r_{i}}{p}, \text { if } n \equiv i \quad(\bmod p) .
$$

(2) A multiplicative Conway $M_{p}$-map corresponds to $M_{p}$-tuples for which $r_{i}=0$, for all $i \in[1, p]$.

Notational Agreement 1 For notational simplicity, we use the same symbol $T$ for all general and special Conway maps, indicating when necessary which particular map, such as Collatz (1) or multiplicative Conway, or anything else, is specifically discussed.

Conway proved in ]52[, ]53[ that multiplicative Conway maps are universal computational devices able to simulate Turing machines; we call such maps, both multiplicative and general, universal Conway maps. Their existence implies that at least for some of the Conway maps the Collatz-type problem is equivalent to the halting problem for a universal Turing machine ]37[ and thus, unsolvable.

Conway's theoretical framework represents the most natural and, one can say, ultimate generalization of the Collatz problem, and his undecidability result is as fundamental to this framework as the Davis-PutnamMatiyasevich solution of Hilbert's tenth problem [8] is to theory of Diophantine equations. Consequently, looking for a positive solution of the Collatz problem, one is entitled to work with its less sweeping generalizations, starting with that of the $(3 m+d)$-extension of the Collatz problem, the $(3 m+d)$-problem for short (]31[, ]34[, ]105[, and, independently, [3]-]22[).

Let $\mathbb{D}$ be the set of natural numbers relatively prime to 6 ; for any $d \in \mathbb{D}$, called the shift number of the map, define 


$$
T=T_{d}: \mathbb{N} \longrightarrow \mathbb{N} ; \quad T=T_{d}(n)=\left\{\begin{array}{cl}
\frac{n}{2} & \text { if } n \text { is even } \\
\frac{3 n+d}{2} & \text { if } n \text { is odd. }
\end{array}\right.
$$

In the above notations $(1), T=T_{1}$ and $T_{d}=T_{M_{2}, R_{2}}(5)$, with $M_{2}=\{1,3\}, R_{2}=\{0,-d\}$. This makes clear how far "below" we are with the $(3 m+d)$-extension of the Collatz map from the complexity of the general Conway case and its universal unsolvability. It does not preclude, of course, the possibility that the $(3 m+d)$-problem and even the original Collatz problem might be still unsolvable for less universal reasons.

Incidentally, the question of what is the minimal $p>2$ which guarantees the existence of universal computational devices $T_{M_{p}, R_{p}}$ (cf. ]121[) remains an open problem - the first one from a series of open problems which relate the arithmetic properties of the definition of a Conway map to the unsolvability of corresponding halting conditions to the pseudo-randomness of the behaviour of trajectories of the corresponding discrete dynamical system (cf. Problems and Observations (1) below).

\section{The Unsolvability Option and Its Far-Reaching Formal and Heuris- tic Ramifications}

One usually quotes (with the first reference apparently traced to ]104[) the well-known, startling remark of the late Paul Erdös concerning the Collatz problem, "Mathematics is not yet ready for such problems," to certificate its exceptional difficulty. But it could be also perceived as a hint at an exceptionally rich mathematical "harvest" which we might expect from the sustained efforts to solve this problem.

This is the point of the following informal theses inspired by a certain vision, as if from an outer space, of the Diophantine equations theory, this mountainous "landscape", with its lovely oases, its deserts, its "Himalayas" challenging skills and perseverance of "Diophantine climbers", and its forbidding, impenetrable "peaks", unsolvable Diophantine equations. We hope that the theoretical and experimental results presented in the following sections will well illustrate the suggestive power of these informal insight.

Thesis 1. Out of the Diophantine Equations Wilderness, with a Lesson: The presence of unsolvability phenomena in a formally complete mathematical framework brings with it the emergence of a great many isolated and, at first glance, mutually unrelated "mountainous islands", i.e., beautiful, deep, rich in consequences, and difficult "local", i.e., problem-oriented theories, with extremely complicated, lengthy proofs, often preceded and accompanied by intricate, bulky computations, of the most important results and with eventual generalizations and unifications of such local theories representing major theoretical breakthrough. Or, for short, the proximity of unsolvability "peaks" leads to enormous problematic variability and related to it (i) abrupt conceptual discontinuity, (ii) novel meaningfullness, (iii) startling logical complexity. Against such a background, (iv) computational experiment becomes a necessary and respected part of investigations, (v) stimulating the development of new powerful, both general and special, problem oriented methods.

Thesis 2. Into the Collatz Wilderness, to Learn the Lesson: There is no doubt that the unsolvability properties of problems related to iterations of number-theoretical functions are by their very iterative, "dynamic" nature much more immediate and explicit than those of "static" by their definition Diophantine problems (whose unsolvability is invariably proved by long and intricate translations into the language of recursive functions). It means that the Collatz-related landscape conceptualized by Conway's formal framework is incomparably more "steep", irregular, unpredictable, and inaccessible than the Diophantine one, testifying in a most unambiguous way to the five aforementioned phenomena: unpredictable variability, pertinence of vast computational experiments, local discontinuity, meaningfulness, complexity. Wanted: formal methods of noticeable power.

To illustrate these local discontinuity, meaningfulness, complexity attributes, let us remind the reader that, back in 1932, Lothar Collatz has written down in his notebook, alongside his (1) map, another - as we call it today - Conway map:

$$
\text { For }\left\{\begin{array}{l}
M_{3}=\{2,4,4\} \\
R_{3}=\{0,1,-1\}
\end{array}\right\} \text { define: } T(n)=T_{M_{3}, R_{3}}(n)=\left\{\begin{array}{rll}
\frac{2 n}{3} & \text { if } n \equiv 0 & (\bmod 3) ; \\
\frac{4 n-1}{3} & \text { if } n \equiv 1 & (\bmod 3) ; \\
\frac{4 n+1}{3} & \text { if } n \equiv 2 & (\bmod 3)
\end{array}\right.
$$

It is easy to verify that $T$ acts on natural numbers as a one-to-one map; namely, it sends bijectively the sets of natural numbers divisible by 3 with the remainders $0,1,2$ onto, respectively, the set of even natural 
numbers, and the sets of odd natural numbers divisible by 4 with the remainders 1 and 3 . The map has 1 as its only fixed point, $T(1)=1$, and the iterations of $T$ starting at 2 and 4 yield cycles of the length two and five, respectively.

On the other hand, with all ultimately cyclic trajectories of bijective maps being cyclic, it remains an open question whether the trajectory starting at 8 is divergent or cyclic - another open Collatz problem. Neither of the known methods used in the studies of the (first) Collatz problem is helpful in elucidating the iterative behaviour of the map $T$. And if true, the divergence of the $T$-trajectory starting at 8 would represent a modest confirmation of the only existing conjecture dealing with general expanding Conway maps: see Section 5 below.

The following definitions formalize the notions of cyclic, ultimatiely cyclic, and divergent trajectories:

Definition 2 (1) For a given Conway map $T=T_{M_{p}, R_{p}}$, the $T$-trajectory and $T$-cycle of length $\ell(\ell-c y c l i c$, for short) starting at $n$ are defined as follows:

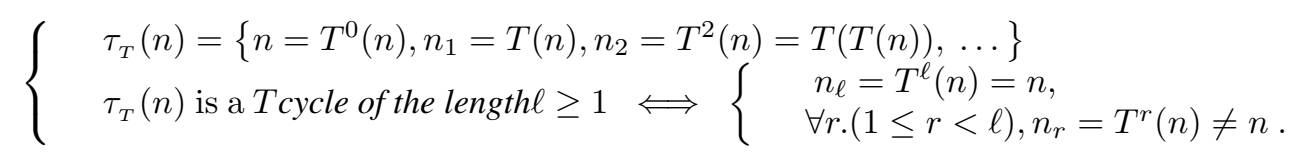

(2) A non-cyclic T-trajectory starting at $n$ is called ultimately $\ell-c y c l i c$ or, for short, ultimately cyclic, if there exist $j \geq 1$ and $\ell \geq 1$, such that the $T$-trajectory starting at $n_{j}=T^{j}(n)$ is $\ell$-cyclic.

(3) A T-trajectory $\tau_{T}(n)$ starting at $n$ is divergent, if it is neither cyclic, nor ultimately cyclic. Or, equivalently, if $\left|T^{j}(n)\right|_{j \rightarrow \infty} \rightarrow \infty$.

(4) We associate with the map $T=T_{M_{p}, R_{p}}$ its contraction-expansion index, or for short, conex-index $\mu(T)=m_{0} \cdots m_{p-1}$.

(5) We call the inequalities $\mu(T)<p^{p}$ and $\mu(T)>p^{p}$ the contraction and, respectively, expansion conditions, and the corresponding Conway maps contracting and expanding, respectively.

\section{The Interplay between the Unsolvability Option for the Gener- alized Collatz Problem and Pseudo-Random Walks in Corre- sponding Dynamical Systems}

To illustrate the above theses, let us look at a very plausible conjecture of Keith R. Matthews and A. M. Watts ]124[, representing, since 1985, the most sweeping generalization of the Collatz conjecture available today.

Conjecture 1 Let $T=T_{M_{p}, R_{p}}$ be a Conway map (4). (i) If $T$ satisfies the contraction condition, $\mu(T)<$ $p^{p}$, then all trajectories $\tau_{T}$ are ultimately cyclic. In particular, there is at least one cycle.

(ii) If $T$ is expanding, $\mu(T)>p^{p}$, then almost all trajectories $\tau_{T}$ are divergent, except possibly for an exceptional set $Q$ of density zero, which means that for all $n$ from $Q$,

$$
\#\{n \in Q ; \mid ;-X \leq n \leq X\}=o(X) .
$$

In particular, there is at least one divergent trajectory.

(iii) The number of cycles is finite.

(iv) If the trajectory $\tau_{T}(n)$ is divergent, then the iterates are uniformly distributed $\left(\bmod p^{q}\right)$ for each $q \in \mathbb{N}$ :

$$
\forall j \in\left[0, p^{q}-1\right], \quad \lim _{N \rightarrow \infty} ; \frac{1}{N+1} ; \#\left\{i \leq N ; \mid ; T^{i}(n) \equiv j \quad\left(\bmod p^{q}\right)\right\}=p^{-q}
$$

Our principal concern lies here with the first, second, and third Matthews-Watts conjectures. An advanced version of the third conjecture limited to $(3 m+d)$-maps will be discussed in Section 6. In the next section, Section 5, we shall exhibit four computational examples confirming the Third Matthews-Watts Conjecture, with the first two confirming the First Conjecture and with the two others confirming the Second Conjecture.

Today, the main heuristic argument in favour of the expected absence (the alternative formulation of the First Conjecture) or presence of divergent trajectories for, respectively, contracting and expanding maps is probabilistic, a particular case of the general approach to Conway dynamical systems (3) - 5] as pseudorandom walks simulated by corresponding Markov chains (cf., e.g., ]112[). This and similar probabilistic heuristics are typically corroborated by empirical data. 
Expected to be implied by the underlying pseudo-random mechanisms, the First Matthews-Watts Conjecture, in its turn, has a striking implication concerning the problem of algorithmic universality of the corresponding maps and unsolvability of related halting problems : cf. Theorem 2 below.

And vice versa, in the case of the Second Conjecture, the algorithmic universality considerations are implying a nontrivial property of dynamical systems produced by universal Conway maps : cf. Theorem 3 below.

The second of the following three theorems is of conditional nature: it recovers algorithmic implications from the first two Matthews-Watts conjectures implied by the character (supposedly, pseudo-random) of walks over Conway-type dynamical systems.

Theorem 1 Conway's unsolvability theorem implies that it is undecidable in the general case whether a trajectory starting at an integer is ultimately cyclic.

Outline of the proof. According to a classic cycle detection device, remarkable for its simplicity, for maps $T$ acting on the set of natural numbers, the trajectory starting at $n \in \mathbb{N}$ is ultimately cyclic iff

$$
\text { for some } k \geq 1, \quad T^{2 k}(n)=T^{k}(n) \text {. }
$$

The undecidability of the verification of the equality (9) for a general Conway map and a given $n$ is an easy corollary of the undecidability of the halting problem for such maps.

Theorem 2 The First Matthews-Watts conjecture implies that contracting Conway maps are not universal.

Outline of the proof. According to the conjecture, all trajectories are ultimately cyclic. Thus, for contracting maps, an equivalent of Turing halting problem can be always resolved. According to Theorem 1 . such maps are not universal.

Theorem 3 The exceptional set $M_{T}$ of a universal (and, thus, according to Theorem 2, expanding) Conway map $T$ is nonempty, infinite, and recursively innumerable.

Outline of the proof. (1) A universal map $T$ can be "pre-programmed" to simulate any other map, including contracting ones and those that have cyclic and ultimately cyclic trajectories. Thus, such a map should have at least one cycle and related ultimately cyclic trajectory or trajectories. Hence, $Q=Q_{T} \neq \emptyset$.

(2) Elements of the set $Q$ are numbers $n \in \mathbb{Z}$ belonging to cyclic or ultimately cyclic $T$-trajectories. $T$ being a universal map, the problem of deciding whether or not $n \in Q$ is unsolvable.

Problems and Observations. (1) In the eventuality Matthews-Watts conjectures are true, Theorem 2 relates the numerical characteristics $p, p^{p}$ and $\mu(T)$ of a Conway map $T=T_{M_{p}, R_{p}}$ to its algorithmic universality and solvability properties. There are not known at present any other numerical criteria for such properties involving the $T$-parameters $p, m_{0}, \ldots, m_{p-1}, r_{0}, \ldots r_{p-1}$. In particular:

(i) What is the minimal value of $p$ yielding a universal Conway map $T=T_{M_{p}, R_{p}}$ ?

(ii) If $T_{1}, T_{2}$ are two Conway maps with the identical parameters $p=p_{1}=p_{2}, \mu=\mu\left(T_{1}\right)=\mu\left(T_{2}\right)$ but two substantially different sets of multiplicative coefficients, $M_{T_{1}} \neq M_{T_{2}}$, ("substantially different" signifies here that they remain different after any permutation of their elements), how this difference affects their dynamical and algorithmic properties ? We present in Section 5 experimental data concerning two substantially different expanding Conway maps with the identical parameters $p$ and $\mu, p=3, \mu=28>$ $p^{p}=27$, but apparently quite different expanding dynamics.

(2) If the Second Matthews-Watts conjecture holds, it would strongly suggest that the unsolvability of the halting problem for Turing machines and of its numerous analogues does not a priori preclude the possibility of our gaining some substantial, if not crucial knowledge about the outcome of related recursive procedures for "almost all" input data.

(3) On the other hand, according to the above Theses 1, 2 (Section 3), the particular methods needed for formally extracting such a knowledge might heavily depend on the formal context of related problems, with the logical complexity and length of corresponding proofs growing with the strength and generality of such problems. 


\section{The First Three Matthews-Watts Conjectures and Their Experi- mental Confirmation}

We have carried a series of about two dozen computer experiments for both contracting and expanding Conway maps, with the parameters $\mu$ chosen to keep to the absolute minimum, respectively, the positive (i.e., guaranteeing the contraction condition) and negative (i.e., guaranteeing the expanding condition) difference between $p^{p}$ and $\mu,\left|p^{p}-\mu\right|=1$.

This choice has the specific purpose of creating "escape" conditions for subsequent iterations, with the expected emergence in the contraction case of very long cycles and even (wishful thinking? cf. our commentary to (12) divergent trajectories, as well as an abundance of ultimate cyclic trajectories in the expanding case.

To simplify calculations without compromising neither their generality, nor the plausibility of our conclusions, we have restricted our choice to only such Conway maps $T$ which preserve the set $\mathbb{N}$ of natural numbers:

$$
T: \mathbb{Z} \longrightarrow \mathbb{Z}, \quad \operatorname{Image}_{\mathrm{T}}(\mathbb{N}) \subset \mathbb{N} \quad T=\left.T\right|_{\mathbb{N}}: \mathbb{N} \longrightarrow \mathbb{N} \text {. }
$$

Our experiments have basically confirmed the first three of Matthews-Watts conjectures.

Experimental study of the First and Third Matthews-Watts Conjectures : The contracting case. Here are two examples of contracting Conway maps:

$$
\left\{\begin{array}{l}
\text { (i) } T_{3}^{(1)}=T_{M_{3}^{1}, R_{3}^{1}}, M_{3}^{1}=\{1,13,2\}, R_{3}^{1}=\{0,1,1\}, \mu\left(T_{3}^{(1)}\right)=26<27=3^{3} ; \\
\text { (ii) } T_{4}=T_{M_{4}, R_{4}}, M_{4}=\{1,17,5,3\}, R_{4}=\{0,1,2,1\}, \mu\left(T_{4}\right)=255<256=4^{4}
\end{array}\right.
$$

(i) $T=T_{3}^{(1)}$. The verification of the conjectures on the interval $n \in \mathbf{I}=\left[1,10^{6}\right]$. One cycle has been recovered, with all other trajectories starting at numbers from the interval $\mathbf{I}$ ultimately entering this cycle:

$$
C_{T_{3}^{(1)}}=\{\min =1, \text { period }=14, \max =186\} .
$$

(ii) $T=T_{4}$. The first Matthews-Watts conjecture has been verified for all natural numbers $n$ less than $10^{6}$, with all trajectories ultimately entering one of the following 7 cycles:

$$
\left\{\begin{array}{l}
C_{T_{4}}^{1}=\{\min =1, \text { period }=2, \max =4\}, \\
C_{T_{4}}^{2}=\{2,1,2\}, \\
C_{T_{4}}^{3}=\{101,37,103227\}, \\
C_{T_{4}}^{4}=\left\{16577,8913,300251686701126925034779159828370513>3 \cdot 10^{35}\right\}, \\
C_{T_{4}}^{5}=\{62210,1332,17543625459549387843430332\}, \\
C_{T_{4}}^{6}=\{198110,452,1532835289361956287\}, \\
C_{T_{4}}^{7}=\{154633,452,66726343095755471773728427\} .
\end{array}\right.
$$

The presence of the very long, extremely far-straying cycle $C_{T_{4}}^{4}$ is due, without any doubt, to the weakness of the contracting condition (10) (ii)).

This experiment has most successfully confirmed the First (and, incidentally, Third) Matthews-Watts Conjecture(s). And, exactly for this reason, it has been a serious disappointment for the authors of the present paper: it did not live up to their expectations to eventually refute the First Matthews-Watts Conjecture by producing strong "escaping" conditions for the emergence of a divergent trajectory in a normatively contracting environment.

Experimental study of the First and Third Matthews-Watts Conjectures : The expanding case. The presence of divergent trajectories considerably complicates experimental studies: in this case, one can be only reasonably, not absolutely sure, following an apparently divergent track even for an extremely long time, that it will not ultimately turn out to be non-divergent.

Here are two examples of expanding Conway maps studied experimentally by us:

$$
\left\{\begin{array}{l}
\text { (i) } T_{3}^{(2)}=T_{M_{3}^{2}, R_{3}^{2}}, M_{3}^{2}=\{1,4,7\}, R_{3}^{2}=\{0,1,2\}, \mu=1 \cdot 4 \cdot 7=28>27=3^{3} ; \\
\text { (ii) } T_{3}^{(3)}=T_{M_{3}^{3}, R_{3}^{3}}, M_{3}^{3}=\{2,2,7\}, R_{3}^{3}=\{0,-1,2\}, \mu=2 \cdot 2 \cdot 7=28>27=3^{3} .
\end{array}\right.
$$


In both cases, we have either traced $T$-trajectories $\tau_{T}(n)$ starting at natural numbers $n$ up to one million, $n \in\left[1,10^{6}\right]$, until they enter a cycle, or have computed their 50000 iterations, with the value of the maximal computed member $n_{T}, \max$ of the trajectory $\tau_{T}(n)$ ranging, in the first case, from $10^{88}$ to $10^{587}$, and in the second case, from $10^{92}$ to $10^{559}$ :

$$
\forall n \in\left[1,10^{6}\right]\left\{\begin{array}{l}
\text { (i) } T=T_{3}^{(2)}, 10^{88}<T^{n_{T}, \max }(n)<10^{587} \\
\text { (ii) } T=T_{3}^{(3)}, 10^{92}<T^{n_{T}, \max }(n)<10^{559} .
\end{array}\right.
$$

(i) $T=T_{3}^{(2)}$. Three $T$-cycles have been recovered:

$$
\left\{\begin{aligned}
C_{T_{3}^{(2)}}^{1} & =\{\min =2, \text { period }=1, \max =2\}, \quad(2 \text { being fixed point }), \\
C_{T_{3}^{(2)}}^{2} & =\{7,11,22\}, \\
C_{T_{3}^{3}}^{3^{(2)}} & =\{10,3,30\} .
\end{aligned}\right.
$$

Following the definition of Matthews-Watts Conjecture 4.2(ii), we associate with the map $T=T_{3}^{(2)}$, two complementary sets of natural numbers, $D$ and $Q$, giving rise, respectively to divergent and convergent trajectories:

$$
\left\{\begin{array}{l}
n \in D_{T} \Longleftrightarrow \tau_{T}(n) \text { is divergent } \Longleftrightarrow \lim _{k \rightarrow \infty} T^{k}(n)=\infty ; \quad D \cup Q=\mathbb{N} ; \\
n \in Q_{T} \Longleftrightarrow \tau_{T}(n) \text { is ultimately cyclic } \Longleftrightarrow \exists N=N(n), \forall k \geq 1 . T^{k}(n)<N .
\end{array}\right.
$$

Our computations show that on the interval $1 \leq n \leq 10^{6}$, the set $Q_{T}$ is much more "massive" than $D_{T}$. However, even on this relatively small interval, the density of $D_{T}$ is steadily growing, in accordance with the prediction of the Second Matthews-Watts Conjecture, as makes clear the below list of 20 numbers, 17p(ii), representing the number of elements of $D_{T_{3}^{(2)}}$ on the 20 consecutive intervals of the length 50000:

$$
\left\{\begin{array}{c}
(\mathbf{i}) \text { If } \mathbf{I}=\left[1,10^{6}\right], \text { then } \#\left(D_{T_{3}^{(2)}} \cap \mathbf{I}\right)=12589 ; \#\left(Q_{T_{3}^{(2)}} \cap \mathbf{I}\right)=987411 \\
(\text { ii) } 182,234,243,240,281,275,333,326,474,643,648 \\
726,739,758,818,1133,1086,1053,1136,1261
\end{array}\right.
$$

(ii) $T=T_{3}^{(3)}$. The data for this, apparently, less volatile case (the effect of the better distributed multiplicative coefficients ? ) are summarized here:

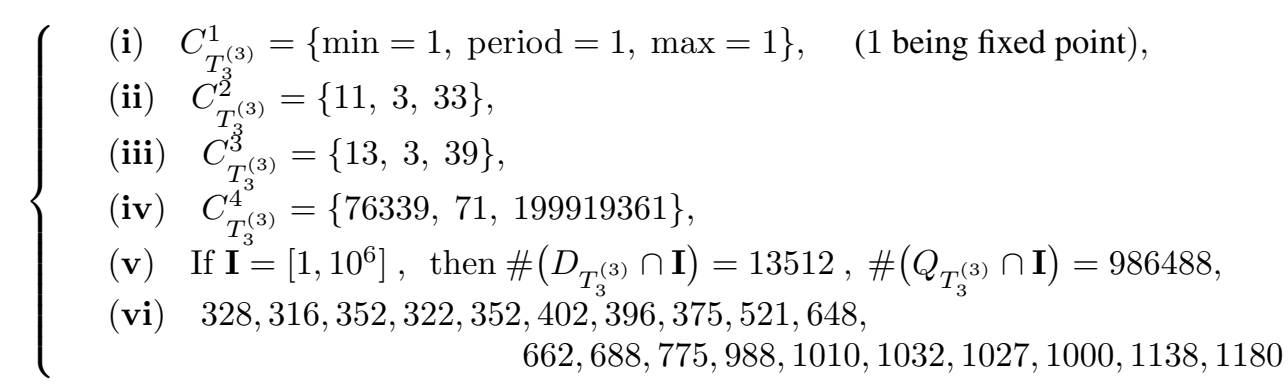

\section{Primitive Cyclic Structure of Dynamical Systems Associated with $(3 m+d)-$ maps}

From the general case for Conway maps, we proceed now to the study of dynamical systems $\mathcal{D}_{d}$ generated by iterations of $(3 m+d)$-functions $T_{d}$ acting, for $d$ relatively prime to $6, d \in \mathbb{D}$, on positive integers according to the algorithm $(6)$. Cyclic structure of $(3 m+d)$-maps is studied here from both theoretical and experimental points of view, with primitive cycles introduced by Jeff Lagarias in 1990 ]105[ being the principal building block of this study.

What we are losing here in the algorithmic generality, we are gaining in the diversity of applicable heuristics and methods, with the Diophantine approximation machinery being a particularly powerful and versatile tool. Its availability is the result of an observation, having a long history, that the walk along the "odd trace" of a cycle of the Collatz dynamical system can be interpreted as a corresponding cyclic transformation of a particularly elegant exponential Diophantine equation preserving some of its characteristics. 
Definition 3 (1) For any integer $r$ and any set of integers $\mathbf{K}$, let $\mathbf{K}_{(r)}$ denote the subset of all $q \in \mathbf{K}$ relatively prime to $r$. In this notation, $\mathbb{D}=\mathbb{N}_{(6)}$.

(2) For a given $d \in \mathbb{D}$, let $T=T_{d}$ be the corresponding map (6), and let, for a given $n \in \mathbb{N}, \tau_{d}(n)=$ $\tau_{T_{d}}(n)$ be, according to $(8)$, the $T_{d}$-trajectory starting at $n$. According to (6) and (8),

$$
q=\operatorname{gcd}(d, n)>1 \quad \Longrightarrow \quad \forall k \geq 1 \cdot q=\operatorname{gcd}\left(d, T_{d}^{k}(n)\right)=\operatorname{gcd}\left(\tau_{d}(n)\right), \tau_{d}(n)=q \cdot \tau_{d / q}(n),
$$

and $\tau_{d}(n)$ is called $q-$ multiple of the $T_{d / q}$-trajectory/cycle $\tau_{d / q}(n)$.

(3) Otherwise, $n \in \mathbb{N}_{(d)}$, and the trajectory $\tau_{d}(n)$ is called primitive, $\operatorname{gcd}(d, n)=\operatorname{gcd}\left(d, T_{d}^{k}(n)\right)=1$, for all positive $k$.

This reduces the study of non-primitive $T_{d}$-cycles to the study of their underlying primitive $T_{q}$-cycles, for all proper divisors $q$ of $d$.

Primitive trajectories are numerous: according to $\sqrt{19}$, the $T_{d}$-trajectory starting at a natural number $n$ relatively prime to $d$ is primitve. But how many of such trajectories are primitive cycles? More specifically, do primitive cycles exist for all systems $\mathcal{D}_{d}, d \in \mathbb{D}$ ? And what are the chances of an integer $n \in \mathbb{D}_{(d)}$ to belong to such a cycle?

These are the subjects of the original conjectures of Lagarias ]105[.

Conjecture 2 (1) Existence of a Primitive $T_{d}$-cycle. For any $d \in \mathbb{D}$, there exists at least one primitive $T_{d}$-cycle. (In the case of the Collatz problem, it is the cycle $\mathbf{C}^{\mathbf{o}}$ (2). Matthews-Watts conjecture 1 i) represents a far-reaching generalization of the present conjecture.)

(2) Finiteness of the Set of Primitive $T_{d}$-cycles. For any $d \in \mathbb{D}$, the number of primitive $T_{d}-$ cycles is finite. (In the case of the Collatz problem, one expects $\mathbf{C}^{\mathbf{0}}$ to be the only primitive cycle. This conjecture, too, finds in Matthews-Watts conjecture 1 iii) its far-reaching generalization.)

In contrast to the first two conjectures of Lagarias, the following conjecture has no immediately apparent $(3 n+1)$ - precursor or analogue:

Conjecture 3 For any $n \in \mathbb{D}$, the set of primitive $T_{d}$-cycles, $d \in \mathbb{D}_{(n)}$, meeting $n$ is infinite.

We interpret this conjecture below as dual to the first conjectures of Lagarias, Conjecture 2, with the emerging disparate duality between $d-$ and $n$-related phenomena being an important new and all-pervading intuition behind the present study. This duality finds its precise formal description in the related Diophantine framework, Theorem 4 (4).

Experimental Study of the Conjectures and Its Cogency: (1) We have systematically studied within the range $1 \leq d \leq 19999, d \in \mathbb{D}$, all 6667 discrete dynamical systems $\mathcal{D}_{d}$ generated by $(3 m+d)$-maps: see [5]. These computations have confirmed two parts of Conjecture 2, albeit inevitably with different degrees of certainty [4]-]23[.

(2) Moreover, the numerical data obtained in our computations suggest the plausibility of previously unknown general laws of the iterative behaviour of $(3 m+d)$-maps: see for detais [4]-]23[. And based on these calculations, the first author has advanced and proved a new deep conjecture concerning a sharp effective upper bound to the minimal member (perigee) of a primitive cycle [Belaga 2003].

(3) Withing the chosen range, Conjecture 2(1) has been fully confirmed: all such systems $\mathcal{D}_{d}$ have at least one primitive cycle.

(4) The confirmation of Conjecture22) is more problematic: a priori, no quantity of computations could confirm that a certain (sub)set is finite. Thus, the plausibility of such a claim hinges on the quality of availble evidence that the search was exhaustive. Such an evidence is discussed in our detailed report [4]-]23[, to justify the claim that in every one of 6667 cases we have localized all existing $(3 m+d)$-primitive cycles:

(i) The total number of such cycles is equal to 42765 .

(ii) The numbers $\omega(d)$ of such cycles for individual systems are ranging from $\omega(d)=1$, for 1481 systems $\mathcal{D}_{d}$ out of 6667 , to 2 , for 1507 of them, to 3 , for 1005 , etc., to 944 for the system $\mathcal{D}_{14303}$. The following table lists eleven systems $\mathcal{D}_{d}$ with the numbers of primitive cycles bigger than 160 :

$$
\begin{aligned}
& d=746318359772715655102899823170211419713085648714303
\end{aligned}
$$

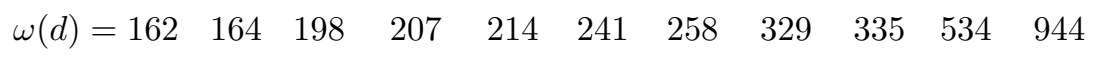


(5) Compared to the above case of Conjecture 2(2), our claim of the confirmation of Conjecture 3 is even more problematic: no quantity of (always finite) computations could confirm that a certain (sub)set is infinite ! Still, taking into account the total number of memberships in primitive cycles of corresponding numbers $n$ ranging from 18 to 452 (for $n=1$ ) - a relatively small for the total number 42765 of primitive cycles found in the chosen range, we have had a strong evidence of the plausibility of Conjecture 3 .

\section{The Shift-Member Disparate Duality}

Our theoretical approach to the cyclic structure of dynamical systems $\mathcal{D}_{d}$ is based on the concepts of primitive membership and disparate duality between related properties of shift and member numbers.

The impetus for this approach comes from the comparison between the above Conjectures 2 and 3 , which is interpreted here as one dual conjecture about projections of the set of memberships. This crucial shift in the perspective becomes possible after the technical passage from primitive cycles to their odd frames, the (obviously, nonempty) cycle of their odd members $n \in \mathbb{D}$.

Definition 4 (1) For any positive integer $n \in \mathbb{N}$, define the number Odd(n) obtained by factoring out of $n$ the highest possible power of 2; thus $\operatorname{Odd}(n)$ is odd and $m=\operatorname{Odd}(n) \cdot 2^{j}$, for some $j$. Hence the notation:

$$
\forall n \in \mathbb{N}, \quad \nu_{2}(n)=\max \left\{j \geq 0 ; \mid ; n \cdot 2^{-j} \in \mathbb{N}\right\}, \quad n=\operatorname{Odd}(n) \cdot 2^{\nu_{2}(n)} .
$$

(2) Let $\tau_{d}(n)=\left\{n, T_{d}(n), T_{d}\left(T_{d}(n)\right), \ldots\right\}$ be a primitive $T_{d}$-trajectory starting at an odd $n$ not divisible by 3 and relatively prime to $d, n \in \mathbf{D}_{(6)}$. The full sequence of odd members, in the order of their appearance in $\tau_{d}(n)$ is called the odd frame of $\tau_{d}(n)$ and denoted by $\operatorname{Oddframe}(n, d)$, with the full sequence of the corresponding exponents of 2, denoted by $\operatorname{Evenframe}(n, d)(21)$ :

$$
\forall n, d \in \mathbb{D}, \quad\left\{\begin{array}{r}
\text { (i) } \tau_{d}(n)=\left\{n, n_{1}, n_{2}, \ldots\right\} \\
\text { (ii) Evenframe }(n, d)=\left\{p_{1}, p_{2}, \ldots\right\} \subset \mathbb{N}, \forall j \geq 1, \\
\quad \forall j \geq 1, p_{j}=\nu_{2}\left(3 n_{j-1}+d\right) \geq 1 ; \\
\text { (iii) } \operatorname{Oddframe}(n, d)=\left\{m_{0}=n, m_{1}, m_{2}, \ldots\right\} \subset \mathbb{D}_{(d)}, \\
\forall j \geq 1, m_{j}=\operatorname{Odd}\left(3 m_{j-1}+d\right)=n_{r_{j}}, r_{j}=\sum_{1 \leq i \leq j} p_{i} .
\end{array}\right.
$$

(3) For a $T_{d}$-cycle $\mathbf{C}=\tau_{d}(n)$ of the length $\ell$, Oddframe $(\mathbf{C})$ is a cycle of the period (oddlength) $k, 1<k<\ell$, satisfying the following obvious inrequality implied by the cyclic condition $(8) i)$ :

$$
\left\{\begin{array}{l}
\text { (i) } \quad \ell=p_{1}+\cdots+p_{k} \geq\left\lceil k \cdot \log _{2} 3\right\rceil \Longleftrightarrow B_{k, \ell}=2^{\ell}-3^{k}>0 \\
\text { (ii) } \Lambda=\left\{(k, \ell) \in \mathbb{N}^{2} \mid \ell \geq\left\lceil k \cdot \log _{2} 3\right\rceil\right\} .
\end{array}\right.
$$

We denote by $\Lambda(23(i i))$ the set of pairs of natural numbers $(k, \ell)$ satisfying the inequality $(23(i))$, and we call $B_{k, \ell}$ the Collatz $(k, \ell)$-number. Number theoretic properties of Collatz numbers are of crucial importance for the behavior of the dynamical systems $\mathcal{D}_{d}$; we have studied the factorizations of Collatz numbers experimentally, in the interval $2 \leq \ell \leq 114$, for all $(k, \ell) \in \Lambda$ : see [6].

(4) Let $\Delta$ be the subset of the set of pairs $(n, d) \in \mathbb{D} \times \mathbb{D}$, with relatively prime integers $n$, $d$. If a primitive $T_{d}$-cycle $\mathbf{C}$ meets a number $n,(n, d) \in \Delta$, then the pair $\langle n, d\rangle$ is called a primitive membership pair, or simply membership. We denote by $\mathcal{P}$ the set of all primitive cycles, by $\mathcal{M}$ the set of all memberships, and, for any $(n, d) \in \Delta$, we define:

$$
\mathcal{M}(n, d)= \begin{cases}\emptyset & \text { if there is no primitive } T_{d}-\text { cycle containing } n, \\ \langle n, d\rangle, & \text { otherwise }\end{cases}
$$

(5) Since, for a given membership $\langle n, d\rangle$, the corresponding primitive $T_{d}$-cycle meeting $n$ is unique, $\mathbf{C}=\tau_{d}(n)$, the set $\mathcal{M}_{n}=\biguplus_{(n, d) \in \Delta} \mathcal{M}(n, d)$ of all memberships with a fixed member number $n$ can be identified with both the set $\mathcal{P}_{n}$ of all primitive cycles meeting $n$ and the $($ sub)set $\mathbb{D}(n)$ of those shift numbers relatively prime to $n$ to which corresponds the primitive $T_{d}$-cycle $\left.\tau_{(} n\right)$ starting at $n$.

(6) On the other hand, the set $\mathcal{M}^{d}=\biguplus_{(n, d) \in \Delta} \mathcal{M}(n, d)$ of all memberships with a fixed shift number $d$ is related to the set $\mathcal{P}^{d}$ of primitive $T_{d}$-cycles by the formula $\# \mathcal{M}^{d}=\sum_{\mathbf{C} \in \mathcal{P}^{d}} \operatorname{Oddlength}(\mathbf{C})$.

Now the Conjectures 2 and 3 could be restated as follows:

Conjecture 4 (1) For any $d \in \mathbb{D}$, the set $\mathcal{M}^{d}$ (or, equivalently, the set $\mathcal{P}^{d}$ ) is nonempty and finite.

(2) For any $n \in \mathbb{D}$, the set $\mathcal{M}_{n}$ (or, equivalently, either of the sets $\mathcal{P}_{n}$ and $\mathbb{D}(n)$ ) is infinite. 
We pass now to the "Diophantization" of the these constructions:

Definition 5 Let $\langle n, d\rangle \in \mathcal{M}$ be a primitive membership. Using the notations (22) and (23), we define:

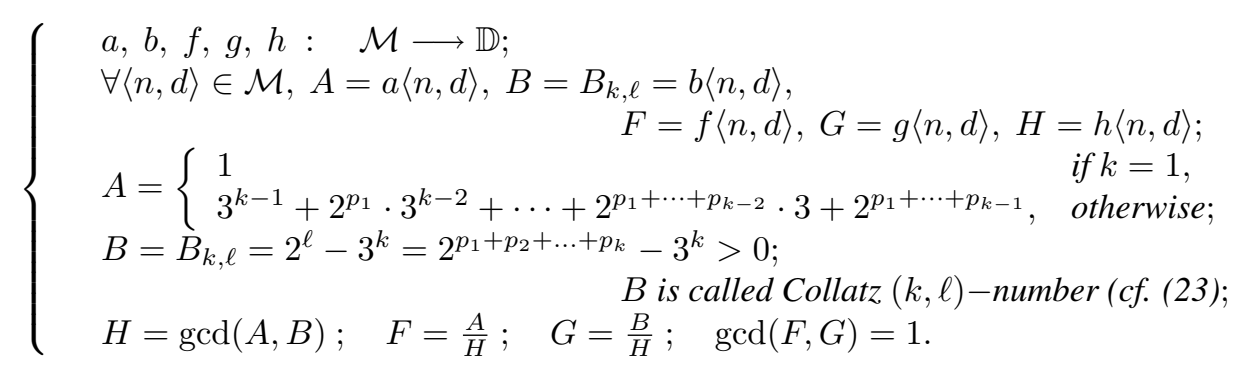

The following theorem translates the iterative "cyclic walk" language for $(3 m+d)$-maps into its Diophantine equivalent:

Theorem 4 (1) Diophantine Cyclic Walk. Let $\langle n, d\rangle \in \mathcal{M}$ be a primitive membership, $\mathbf{C}=\mathbf{C}(d, n)$ be the corresponding primitive $T_{d}-$ cycle starting at $m_{0}=n$, of the oddlength $k>1$, let $m_{1}=\operatorname{Odd}\left(3 m_{0}+d\right)$ (23), and let $\left\langle m_{1}, d\right\rangle$ be the corresponding membership. Let $\sigma_{k}$ be the circular counterclockwise permutation on $k$-tuples of positive integers $\mathbf{P}=\operatorname{Evenframe}(n, d)$. Then the set $\mathbf{P}_{\mathbf{1}}=\operatorname{Evenframe}\left(m_{1}, d\right)$ corresponding to the membership $\left\langle m_{1}, d\right\rangle$ is defined by the formula:

$$
\mathbf{P}_{1}=\operatorname{Evenframe}\left(m_{1}, d\right)=\sigma_{k}(\mathbf{P})=\sigma_{k}\left(p_{1}, p_{2}, \ldots, p_{k-1}, p_{k}\right)=\left(p_{2}, p_{3}, \ldots, p_{k}, p_{1}\right) .
$$

(2) From Primitive Membership to its Diophantine Representation. Let $\langle n, d\rangle$ be a membership, and let the functions $a, b, f, g, h$ be defined as above (22). Then $F=f(\langle n, d\rangle)=n, G=g(\langle n, d\rangle)=d$.

(3) From the Diophantine Formulae to their Membership Interpretation. Let $k \geq 2$ and $\mathbf{P}=$ $\left(p_{1}, \ldots, p_{k}\right) \subset \mathbb{N}^{k}$ be a $k$-tuple of positive integers satisfying the inequality

$$
\ell=|\mathbf{P}|=p_{1}+\cdots+p_{k} \geq\left\lceil k \cdot \log _{2} 3\right\rceil,
$$

and let the numbers $A, B, F, G, H$ be defined as in 22). Then $\langle F, G\rangle$ is a primitive membership.

\section{Why the Diophantine Interpretation of the Collatz and Lagarias Conjectures Strengthen Their Plausibility}

We will need a more descriptive presentation of the above diophantine formulae for primitive memberships:

Definition 6 (1) The pair $(k, \ell) \in \Lambda$ and the corresponding Collatz number $B=B_{k, \ell}(23)$ are called simple if $\operatorname{gcd}(k, \ell)=1$; they are called narrow if $\ell=\left\lceil k \cdot \log _{2} 3\right\rceil$. We denote by, respectively, $\hat{\Lambda}, \Lambda_{0}, \hat{\Lambda}_{0}$, the sets of simple, narrow, and simple narrow pairs $(k, \ell)$ :

$$
\hat{\Lambda}=\{(k, \ell) \in \Lambda \mid \operatorname{gcd}(k, \ell)=1\}, \Lambda_{0}=\left\{(k, \ell) \in \Lambda \mid \ell=\left\lceil k \cdot \log _{2} 3\right\rceil\right\}, \hat{\Lambda}_{0}=\hat{\Lambda} \cap \Lambda_{0} .
$$

(2) Let $\langle n, d\rangle \in \mathcal{M}$ be a primitive $(k, \ell)$-membership with the corresponding Collatz number $B$. We associate with $\langle n, d\rangle \in \mathcal{M}$ its Collatz corona $\mathcal{A}=\mathcal{A}_{k, \ell}$, a finite set of natural numbers depending only on the Oddlength and length of $\langle n, d\rangle \in \mathcal{M}$, as follows:

(a) If $k=1$, then

$$
\mathcal{A}_{1, \ell}=\{1\} .
$$

(b) If $k>1$, then for any aperiodic $(k-1)$-tuple $\mathbf{P}=\left\{p_{1}, \ldots, p_{k-1}\right\}$ of positive integers satisfying the inequality 25,

$$
p_{1}+\ldots+p_{k-1}<\ell
$$

the following number belongs to $\mathcal{A}$ :

$$
A=A(\mathbf{P})=3^{k-1}+3^{k-2} \cdot 2^{p_{1}}+\ldots+3 \cdot 2^{p_{1}+\ldots+p_{k-2}}+2^{p_{1}+\ldots+p_{k-1}} .
$$

By definition, members of Collatz corona are odd positive integers not divisible by 3.

(3) The Collatz corona corresponding to a simple, narrow, or simple narrow pair $(k, \ell)$ is called, respectively, simple, narrow, simple narrow. 
Theorem 5 (1) Collatz corona $\mathcal{A}_{k, \ell}$ is a one-element set iff $k=1$ (28).

(2) Otherwise, Collatz corona $\mathcal{A}_{k, \ell}$ is a finite set of mutually distinct positive integers not equal to he Collatz number $B=B_{k, \ell}$, and its cardinality $\alpha_{k, \ell}=\# \mathcal{A}_{k, \ell}$ is given by the following formula:

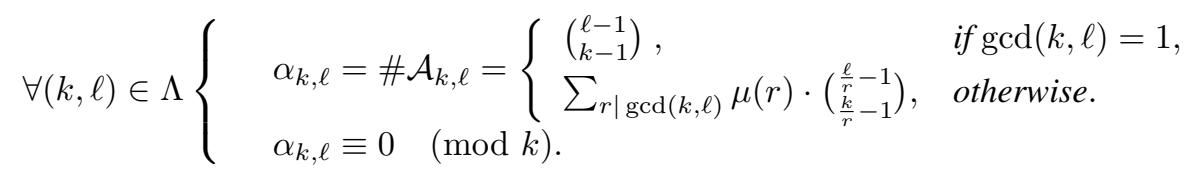

where $\mu$ is the Möbius function:

$$
\mu(m)= \begin{cases}1 & \text { if } m=1, \\ (-1)^{q} & \text { if } m \text { is the product of } q \text { distinct primes } \\ 0, & \text { if } m \text { is divisible by a square of a prime. }\end{cases}
$$

The low part of the formula 31 , defined for pairs $(k, \ell)$ with the property $\operatorname{gcd}(k, \ell)>1$, is universal and covers - but also obscures - the simple upper case $\operatorname{gcd}(k, \ell)=1$.

(3) The below lower and upper bounds to members A of Collatz corona are sharp:

$$
\left(\begin{array}{l}
\forall(k, \ell) \in \Lambda \\
\forall A \in \mathcal{A}_{k, \ell}
\end{array}\right)\left\{\begin{array}{l}
3^{k-1}<\underline{A}_{k, \ell} \leq A \leq \bar{A}_{k, \ell}<2^{\ell-k+1} \cdot 3^{k-1} \\
\underline{A}_{k, \ell}=3^{k}-2^{k}, \bar{A}_{k, \ell}=2^{\ell-k+1} \cdot\left(3^{k-1}-2^{k-1}\right)+3^{k-1} .
\end{array}\right.
$$

(4) Since the function $a$ in the definition (25) does not actually depend on the $k$-th component $p_{k}$ of the correspnding Collatz configuration, its values for two $k$-configurations of different lengths can be equal. More precisely,

$$
\forall(k, \ell) \in \Lambda \quad \forall j \geq 1, \quad \mathcal{A}_{k, \ell} \subset \mathcal{A}_{k, \ell+j} .
$$

(5) With the exception of the trivial case, corresponding to the primitive $T_{1}$-cycle $\mathbf{C}^{0}$ (cf. (2) and the above statement (1)),

$$
k=1, \ell=2, \mathcal{A}_{1,2}=\{1\}, B=1,
$$

no Collatz number ever belongs to the respective Collatz corona and, if $k \geq 4$, it is located below the upper bound (33) of the corona,

$$
\forall(k, \ell) \in \Lambda, B=B_{k, \ell}<2^{\ell}\left(<\bar{A}_{k, \ell}, \text { if } k \geq 4\right) .
$$

(6) Moreover, if $(k, \ell)$ is not narrow, then $B$ is located above the low bound (33) of the corona:

$$
\forall k \geq 1 .(k, \ell) \in \Lambda \backslash \Lambda_{0} \Longrightarrow B=B_{k, \ell}=2^{\ell}-3^{k}>\underline{A}_{k, \ell}=3^{k}-2^{k} .
$$

This is no more the case, if $(k, \ell)$ is narrow:

$$
\forall k \geq 1 .(k, \ell) \in \Lambda_{0} \Longrightarrow B=B_{k, \ell}=2^{\ell}-3^{k}<\underline{A}_{k, \ell}=3^{k}-2^{k} .
$$

(7) The original, $3 n+1$ Collatz conjecture is equivalent to the following claim: for any nontrivial pair $(k, \ell) \in \Lambda \backslash\{(1,2)\}$, no member of the Collatz corona $\mathcal{A}_{k, \ell}$ is devisible by the Collatz number $B_{k, \ell}$.

(8) The first conjecture of Lagarias is equivalent to the following claim: for any $d \in \mathbb{D}$, there exists at least one and not more than a finite number of pairs $(k, \ell) \in \Lambda$, such that, for some number from the $(k, \ell)$-corona, the equality holds

$$
d=\frac{B_{k, \ell}}{\operatorname{gcd}\left(B_{k, \ell}, A\right)}, \quad A \in \mathcal{A}_{k, \ell}
$$

(9) The second conjecture of Lagarias is equivalent to the following claim: for any $n \in \mathbb{D}$, there exist an infinite number of pairs $(k, \ell) \in \Lambda$, such that, for some number from the $(k, \ell)$-corona, the equality holds

$$
n=\frac{A}{\operatorname{gcd}\left(B_{k, \ell}, A\right)}, \quad A \in \mathcal{A}_{k, \ell} .
$$


Why and how the formulae (38), (39) strengthen the plausibility of Collatz and Lagarias conjectures. Collatz Conjecture: According to Theorem 577), one needs to prove that, for any $(k, \ell) \in \Lambda \backslash\{(1,2)\}$, the Collatz number $B=2^{\ell}-3^{k}$ divides neither of a finite number (cf. 31) of members $A$ of its corona $\mathcal{A}_{k, \ell}$. Lagarias First Conjecture: A similar observation : cf. (38)

Lagarias Second Conjecture: (1) In the case $n=1$, the Diophantine version of the conjecture asserts (cf. (39)) that there exists an infinite number of pairs $\{A, B\}, B$ being a Collatz number, $A$ being a member of the corresponding Collatz corona, such that $A$ divides $B$. Contrary to the above reasoning, the change of the places of the actors, $A$ and $B$, brings with it an infinite number of possibilities: if $A \in \mathcal{A}_{k, \ell}$, then, according to the the inclusion (34, $, A \in \mathcal{A}_{k, \ell+j}, \forall j \geq 1$, which means that one can look for $B$ divisible by $A$ among an infinite number of candidates $B_{k, \ell+j}$.

A similar argument holds in the general case $n \in \mathbb{D}:$ cf. (39)

Acknowledgements. (1) The authors are grateful to Keith Matthews, of Brisbane, Australia, and Thomàs Oilvera et Silva, of Aveiro, Portugal, for their important corrections and suggestions.

(2) The authors are grateful to two anonymous referees for their helpful reports.

(3) The first author is grateful:

(i) to Mme Christine Disdier, - for her enduring, unflinching support at the Strasbourg Mathematical Institute's library;

(ii) to his colleagues of the First International Conference on the Collatz Problem, at the Katholische UniversitÉt, EichstÉtt, Germany, 1999, - for continuing fruitful discussions;

(iii) to Mlle Véronique Bertrand, - for her indispensable help in the preparation of the Web site for the (unfortunately, indefinitely adjourned) Second International Conference on the Collatz Problem, at the Université Louis Pasteur, Strasbourg, France;

(iv) and to his son Abraham, - for his interest and help in discussing the first version of the present paper.

\section{References}

[1] E. G. Belaga, Probing into the $3 m+d$ World, Preprint 1995, math. 95/03, Univ. Louis Pasteur, Strasbourg.

[2] E. G. Belaga, Reflecting on the $3 m+1$ Mystery. Outline of a Scenario - Improbable or Realistic ? Preprint 1998, math. 98/49, Univ. Louis Pasteur, Strasbourg. Available online at http://www-irma.ustrasbg.fr/irma/publications/1998/98049.shtml

[3] E. G. Belaga, M. Mignotte, Embedding the $3 m+1$ Conjecture in a $3 m+d$ Context, Experimental Mathematica 7:2 (1999) 145-151.

[4] E. G. Belaga, M. Mignotte, Cyclic Structure of Dynamical Systems Associated with $3 m+d$ Extensions of Collatz Problem, Preprint 2000, math. 2000/17, Univ. Louis Pasteur, Strasbourg. Available online at http://www-irma.u-strasbg.fr/irma/publications/2000/00017.shtml

[5] E. G. Belaga, M. Mignotte, The Collatz Problem and Its Generalizations: Experimental Data. Table 1. Primitive Cycles of $(3 m+d)$-mappings, Preprint 2006, math. 2006/15, Univ. Louis Pasteur, Strasbourg. Available online at http://www-irma.u-strasbg.fr/irma/publications/2006/06015.shtml

[6] E. G. Belaga, M. Mignotte, The Collatz Problem and Its Generalizations: Experimental Data. Table 2. Factorization of Collatz Numbers $2^{\ell}-3^{k}$, Preprint 2006, math. 2006/16, Univ. Louis Pasteur, Strasbourg. Available online at http://www-irma.u-strasbg.fr/irma/publications/2006/06016.shtml

[7] J. Lagarias, $3 x+1$ Problem: Annotated Bibliograph, arxiv.org/math.NT/0309224 (the last update: January 5,2006 ).

[8] J. P. Jones, Yu. V. Matijasevic, Proof of Recursive Unsolvability of Hilbert's Tenth Problem, Amer. Math. Monthly 98 (1991) 689-709. 